\title{
Comparison of gram-negative and gram-positive hematogenous pyogenic spondylodiscitis: clinical characteristics and outcomes of treatment
}

\author{
Ching-Yu Lee, ${ }^{1,5,7}$, Meng-Huang Wu ${ }^{6,7}$, Chin-Chang Cheng ${ }^{1,5,7}$, Tsung-Jen Huang ${ }^{6}$, Tsung-Yu Huang ${ }^{2}$,
} Chien-Yin Lee ${ }^{1}$, Jou-Chen Huang ${ }^{3,4}$ and Yen-Yao Li ${ }^{1,5^{*}}$

\begin{abstract}
Background: To the best of our knowledge, no study has compared gram-negative bacillary hematogenous pyogenic spondylodiscitis (GNB-HPS) with gram-positive coccal hematogenous pyogenic spondylodiscitis (GPC-HPS) regarding their clinical characteristics and outcomes.

Methods: From January 2003 to January 2013, 54 patients who underwent combined antibiotic and surgical therapy in the treatment of hematogenous pyogenic spondylodiscitis were included.

Results: Compared with 37 GPC-HPS patients, the 17 GNB-HPS patients were more often found to be older individuals, a history of cancer, and a previous history of symptomatic urinary tract infection. They also had a less incidence of epidural abscess formation compared with GPC-HPS patients from findings on magnetic resonance imaging (MRI). Constitutional symptoms were the primary reasons for initial physician visits in GNB-HPS patients whereas pain in the affected spinal region was the most common manifestation in GPC-HPS patients at initial visit. The clinical outcomes of GNB-HPS patients under combined surgical and antibiotic treatment were not different from those of GPC-HPS patients. In multivariate analysis, independent predicting risk factors for GNB-HPS included a malignant history and constitutional symptoms and that for GPC-HPS was epidural abscess.
\end{abstract}

Conclusions: The clinical manifestations and MRI presentations of GNB-HPS were distinguishable from those of GPC-HPS.

Keywords: Gram-negative infection, Gram-positive infection, Hematogenous pyogenic spondylodiscitis

\section{Background}

Hematogenous pyogenic spondylodiscitis (HPS) is an infection involving the intervertebral disc and adjacent vertebrae that occurs by hematogenous spread of bacteria from a distant site. HPS is highly associated with diabetes mellitus, infective endocarditis, chronic kidney disease, cancer, immunosuppressive disorders, and intravenous drug abuse. The prevalence of HPS is more frequent in males and the elderly $[1,2]$. The main

\footnotetext{
* Correspondence: orthoejaca@gmail.com

'Department of Orthopedic Surgery, Chang Gung Memorial Hospital, No. 6, West Sec., Chia Pu Rd., PuTz, Chiayi, Taiwan

${ }^{5}$ College of Medicine, Chang Gung University, Taoyuan, Taiwan

Full list of author information is available at the end of the article
}

causative microorganisms include gram-positive cocci (GPC), especially Staphylococcus aureus, which account for $40-60 \%$ of HPS patients [2, 3], and gram-negative bacilli (GNB) which constitute $15-23 \%$ of cases $[2,4]$. Despite the significant incidence of HPS caused by GNB, few studies in the literature are concerned with the clinical characteristics and outcomes of hematogenous spinal infections caused by GNB $[5,6]$.

To the best of our knowledge, no reports have compared the clinical characteristics and outcomes of HPS caused by GNB vs. GPC. The goal of this study was to evaluate clinical presentations, past infectious histories, imaging findings, and clinical outcomes in GNB-HPS vs. GPC-HPS patients. 


\section{Methods}

\section{Patients}

From January 2003 to January 2013, a retrospective review of 54 patients with HPS was performed using the Spine Operation Registry of our institution. All patients included in this study underwent combined antibiotic and surgical treatment. The indications for surgical treatment included poor response to antibiotic therapy, worsening neurologic impairment, epidural abscess formation, and/or significant osseous destruction with spinal instability. Surgical approaches included anterior or posterior spinal surgery, or combined anterior and posterior approaches. Because the clinical and radiological presentations differed among the patients (Figs. 1 and 2), the surgical approach was chosen based on the individual patient's presentation, i.e., the level of the involved vertebrae, the extent of osseous destruction, and the presence or absence of an abscess and its location, if present.

All HPS patients received a 3-month course of antibiotic therapy which consisted of at minimum 2 week course of parenteral antibiotics based on culture results. Outpatient oral antibiotic treatment completed a 3 month course of antibiotics after normalization of serum $\mathrm{C}$-reactive protein (CRP) levels and leukocyte counts. Patients who survived were followed-up for a minimum of 2 years.

Pyogenic spondylodiscitis was defined as a spinal infection encompassing both vertebral osteomyelitis and discitis [7]. The definitive diagnosis of pyogenic spondylodiscitis was based on clinical presentation, imaging findings from plain radiographs and contrast-enhanced magnetic resonance imaging (MRI), and intraoperative bacteriologic cultures [4]. Exclusion criteria were: (1) negative microbiological culture from the infected specimen; (2) non-pyogenic infection; (3) surgical site infection associated with spine surgery and percutaneous spinal procedures including epidural steroid injection, selective nerve root block, and radiofrequency ablation; (4) history of previous spinal surgery on any level; and (5) history of a bedsore or penetrating wound on the back.

\section{Data assessment}

In order to compare the clinical manifestations and outcomes between GNB-HPS and GPC-HPS patients, patient characteristics, underlying comorbidities, bacteriologic results, MRI findings, and final outcomes were reviewed using the electronic database at our hospital. The imaging findings were reviewed independently by two spine surgeons (CCC, and YYL) who were unaware of the culture results. For a history of an infectious disease, the patient sustained an infectious disease before pyogenic spondylodiscitis and received antimicrobial therapy. The history of symptomatic urinary tract infection (UTI) would be recorded when a patient was admitted for parenteral antibiotics therapy to treat cultured-proved urinary tract infection. The history of infectious diseases has been completely recorded and reviewed in every patient's electronic chart.

A one-level spinal infection was defined as an infection involving two contiguous vertebral bodies and one adjacent intervertebral disc, and a two-level spinal infection was involving three contiguous vertebral bodies and two adjacent discs.

A spinal epidural abscess (Fig. 3) was defined as an epidural mass with iso-or hypointensity on T1-weighted
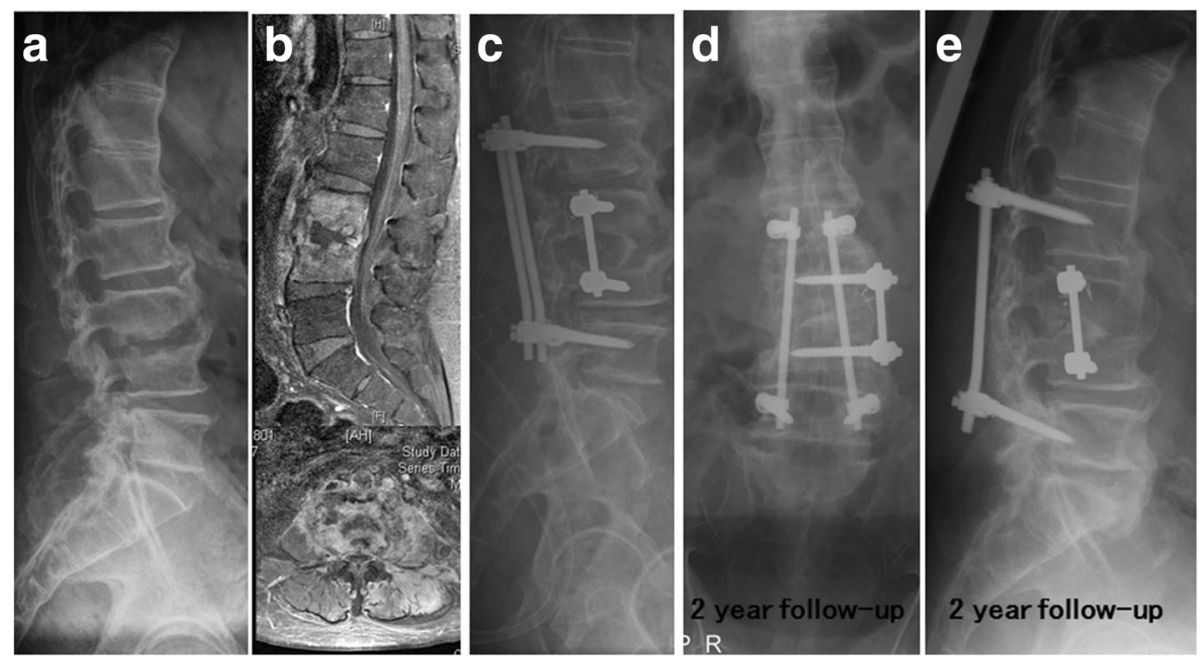

Fig. 1 Escherichia coli infectious spondylodiscitis of L3-4 in a patient with a past history of colon cancer (a). Disc space was narrowing with nearby destructed endplates on L3-4 level; $\mathbf{b}$ Osteomyelitis in L3-4 vertebral bodies, discitis and psoas abscess without epidural abscess were found in gadolinium-enhanced magnetic resonance imaging; c Anterior and posterior spinal surgery was noticed in the immediate postoperative X-ray.; $\mathbf{d}$ and $\mathbf{e}$ Solid bone fusion was noticed at the 2-year follow-up 

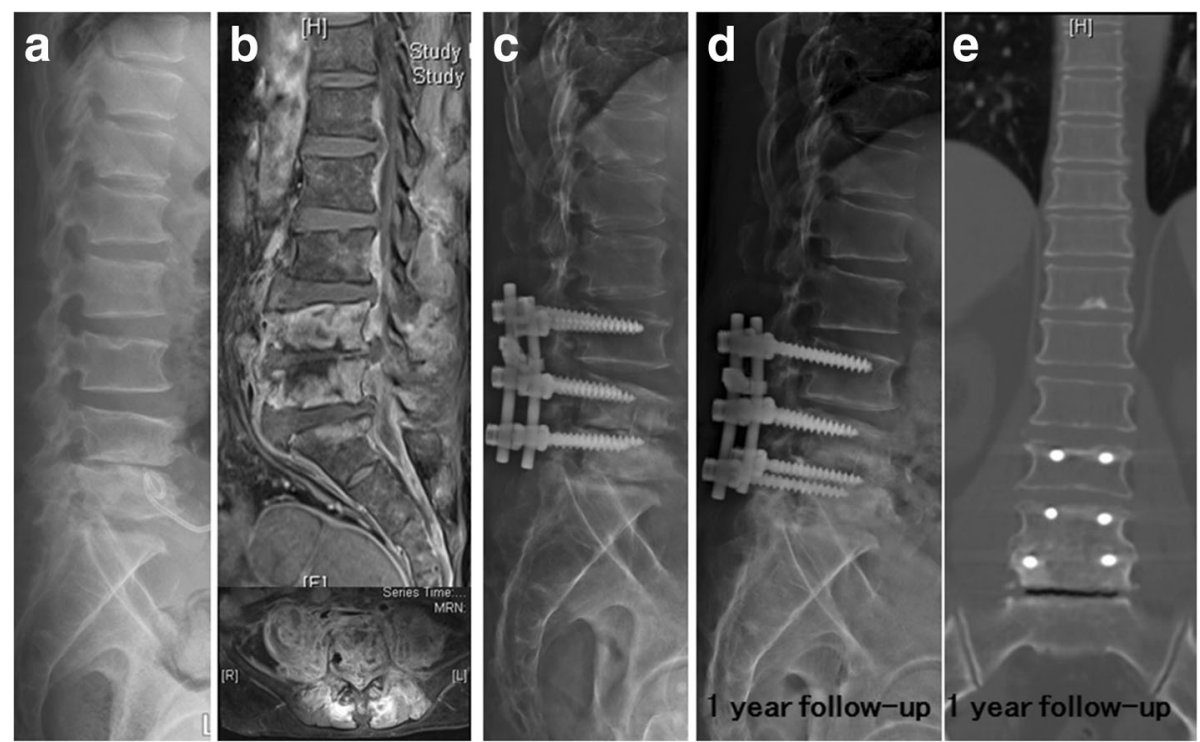

Fig. 2 Hematogenous Pyogenic Spondylodiscitis of L4-5 due to methicillin-resistant Staphylococcus aureus infections (a). Narrowing disc space with endplate erosion with pigtail catheter placement for drainage of paravertebral abscess was noticed on L4-5 level; $\mathbf{b}$ Abscess formation was found in ventral epidural space, bilateral psoas and back muscles; c Supplementary posterior spinal instrumentation was performed 6 weeks after anterior debridement and a tricortical iliac strut bone graft for intervertebral fusion; $\mathbf{d}$ and e Solid bone fusion on L4-5 was noticed at the 1 -year follow-up

images, hyperintensity on T2-weighted images and linear enhancement surrounding non-enhancing purulent or necrotic matter on MRI [8].

To assess clinical outcomes following combined antibiotic and surgical treatment, delayed wound healing was defined as prolonged wound exudate, or persistent fever with erythematous wound two weeks after administration of antibiotics and surgical treatment, relapsing infection was defined as recurrent spinal infection within 1 year after resolution of infection at hospital discharge. Mortality was defined as death owing to progressive sepsis or medical complications.

\section{Statistical methods}

Univariate analysis was used to determine factors associated with GNB-HPS and GPC-HPS. An independent student $t$ test was used for numerical data. A $X 2$ analysis or a Fisher's exact test was used for categorical data. Descriptive data were presented as the mean with standard deviation for quantitative variables and as frequency for categorical variables. Statistical significance was set at a $p$-value of $<0.05$. Multivariate analysis was used to determine independent predicting factors associated with GNB-HPS and GPC-HPS. All factors showing statistical significance ( $p$-value of $<0.05)$ in univariate analysis were incorporated in multivariate logistic regression analysis. Statistical analyses were performed using the Statistical Package for the Social Sciences for Windows (SPSS, version 12.0).

\section{Results}

\section{Patient characteristics}

A total of 54 HPS patients included 17 GNB-HPS patients and 37 GPC-HPS patients (Table 1). The average follow-up period was 4.5 years (range, 2-6 years). GNBHPS patients were significantly older than GPC-HPS patients (mean age, 65 vs. 55 years, respectively; $p=0.014$ ).

With regards to symptoms at initial visit, only back pain predominated in GPC-HPS (73\%) compared with GNB-HPS patients (30\%), and the prevalence of constitutional symptoms (fever or drowsiness) with/without back pain were more frequent in GNB-HPS patients ( $70 \%$ vs. $27 \%, p=0.003$ ). The average duration of symptoms in GNB-HPS vs. GPC-HPS patients were 18 vs. 37 days, respectively $(p=0.162)$.

Seven of 17 GNB-HPS patients had a history of malignancy, including three colorectal cancer patients, one cervical cancer patent, one lung cancer patient, one breast cancer patient, and one bladder cancer patient. One GPC-HPS patient had a history of hypopharyngeal cancer. A history of malignancy was more commonly represented in GNB-HPS compared with GPC-HPS patients $(41 \%$ vs. $3 \%$, respectively; $p<0.001)$.

Delayed wound healing was found in eight patients (one patient with GNB infection and seven patients with GPC infection); relapse of infection occurred in eleven patients (one patient with GNB infection and ten patients with GPC infection); eight deaths occurred in the HPS patients including three deaths in GNB-HPS 

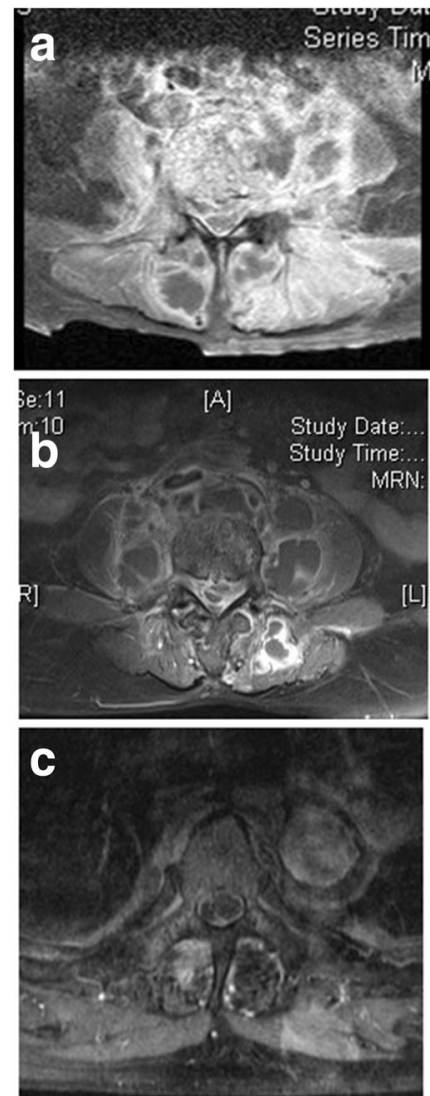
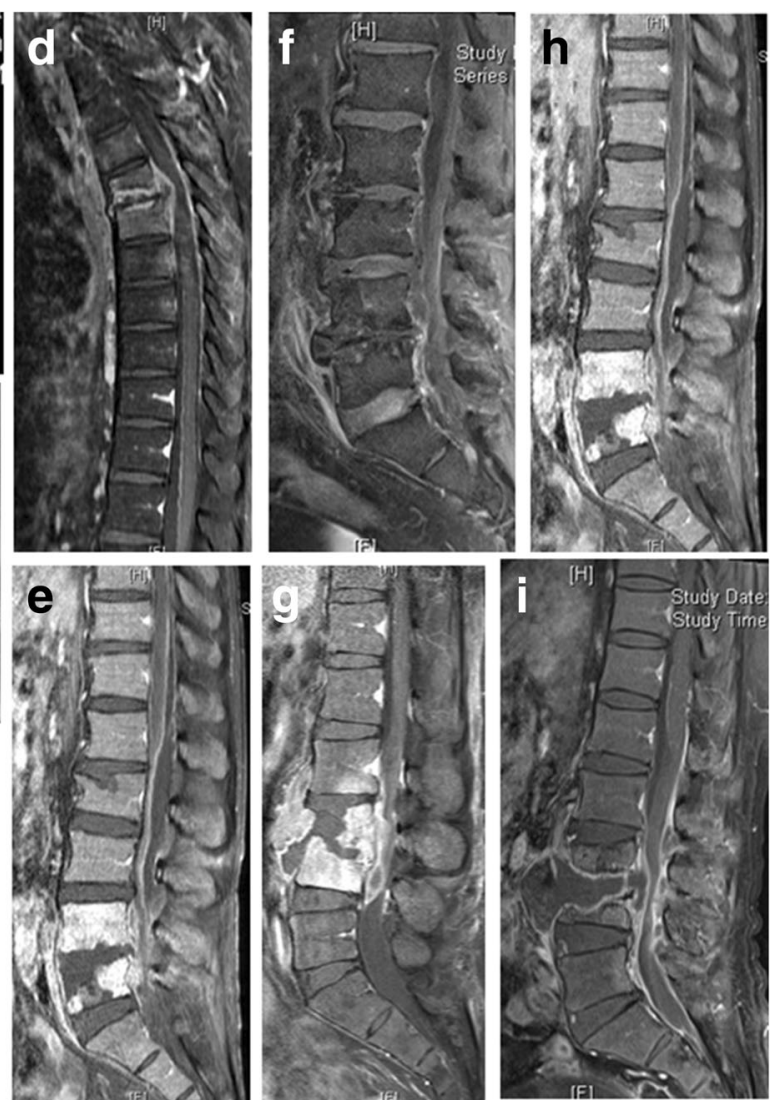

Fig. 3 Elucidation of epidural abscess and muscle abscess in contrast-enhanced magnetic resonance imaging with fat suppression among 9 g-positive pyogenic spondylodiscitis $(\mathbf{a}-\mathbf{c})$. The spinal epidural abscess was defined as an epidural mass with iso-or hypointensity on T1-weighted images, which was surrounded by linear enhancement (ring sign) on magnetic resonance imaging. Abscess formation in psoas muscle or back muscle was defined as an asymmetrical enlarged mass of the involved muscle with ring sign on magnetic resonance imaging. $\mathbf{d}$-f $\mathbf{f}$ Ventral epidural abscess was noticed on sagittal gadolinium-enhanced fat-suppressed T1-weighted magnetic resonance

patients and five deaths in GPC-HPS patients. Among the eight deaths, six patients died of uncontrolled infection, and two patients died of comorbidities within 3 months after hospital discharge (one from liver cirrhosis (Child-Pugh Class B) and upper gastrointestinal bleeding, and one from complicated pneumonia). All ten patients with relapsing infection had successful resolution of infection after a second surgical debridement and completion of antibiotic therapy. There were no significant differences in relapse of infection or mortality between GNB-HPS and GPC-HPS patients. All of survival patients with eradication of infection had solid fusion in spondylodiscitis at the final radiographic follow-up ( $p=0.696)$.

\section{History of infectious disease}

Twenty-three (43\%) of 54 patients had histories of previous infections, including 7 (41\%) GNB-HPS patients and 16 (43\%) GPC-HPS patients (Table 2). GNB-HPS patients had a significantly higher frequency of previous symptomatic UTI compared with GPC-HPS patients (41\% vs. $3 \%$, respectively; $p=0.03$ ). There was no significant difference in histories of other infectious disorders.

\section{Microbiologic findings}

The microbiologic results from infected intraoperative specimens in the 54 HPS patients are outlined in Table 3. Among the GNB-HPS patients, Escherichia coli was the most commonly isolated pathogen, involved six patients (35\%), followed by Enterobacter cloacae in four (24\%) patients, Klebsiella pneumonia in two (12\%) patients, Salmonella enteric in two (12\%) patients, Pseudomonas aeruginosa in two patients (12\%), Proteus mirabilis in one $(6 \%)$ patient, and Citrobacter freundii in one (6\%) patient. For GPC-HPS, Staphylococcus aureus was the most common isolate (86\%). The other isolated GPC pathogens were Coagulase-negative staphylococcus (14\%), and Streptococcus sp. (8\%). All organisms cultured from intraoperative infected specimens were the same as those obtained from earlier blood cultures. 
Table 1 Characteristics of 54patients with hematogenous pyogenic spondylodiscitis

\begin{tabular}{|c|c|c|c|}
\hline variable & GNB $(n=17)$ & $\mathrm{GPC}(n=37)$ & $p$ \\
\hline$\overline{\text { Age }(y r)}$ & $65 \pm 14.2$ & $55 \pm 14.5$ & $0.014^{*}$ \\
\hline Male sex (No. [\%]) & $11(65)$ & $31(84)$ & 0.162 \\
\hline Follow-up (yr) & $4.8(2-6)$ & $4.4(2-6)$ & 0.372 \\
\hline \multicolumn{4}{|l|}{ Affected level (No. [\%]) } \\
\hline 1 & $14(82)$ & $28(76)$ & \multirow[t]{2}{*}{0.732} \\
\hline 2 & $3(18)$ & $9(24)$ & \\
\hline \multicolumn{4}{|l|}{ Location of spinal lesions (No. [\%]) } \\
\hline Cervical spine & 0 & $2(5)$ & \multirow[t]{3}{*}{0.601} \\
\hline Thoracic spine & $2(12)$ & $5(14)$ & \\
\hline Lumbosacral spine & $15(88)$ & $30(81)$ & \\
\hline \multicolumn{4}{|c|}{ Symptoms at the initial visit (No. [\%]) } \\
\hline Back/neck pain only & $5(30)$ & $27(73)$ & \multirow[t]{3}{*}{$0.003^{*}$} \\
\hline Constitutional symptoms only & $6(35)$ & $2(5)$ & \\
\hline $\begin{array}{l}\text { Back pain with constitutional } \\
\text { symptoms }\end{array}$ & $6(35)$ & $8(22)$ & \\
\hline Duration of symptoms (day) & $18 \pm 21.8$ & $37 \pm 51.6$ & 0.162 \\
\hline \multicolumn{4}{|l|}{ Comorbidity (No. [\%]) } \\
\hline Diabetes mellitus & $4(24)$ & $18(49)$ & 0.135 \\
\hline $\begin{array}{l}\text { End stage renal failure with } \\
\text { hemodialysis }\end{array}$ & 0 & $5(30)$ & 0.168 \\
\hline Chronic liver disease & $6(35)$ & $12(32)$ & 0.824 \\
\hline Malignancy & $7(41)$ & $1(3)$ & $<0.001^{*}$ \\
\hline Intravenous drug abuse & 0 & $6(16)$ & 0.161 \\
\hline Neurologic symptoms (No. [\%]) & $12(71)$ & $22(59)$ & \multirow[t]{4}{*}{0.432} \\
\hline Radicular pain & $9(53)$ & $13(35)$ & \\
\hline Limb weakness & $7(41)$ & $11(30)$ & \\
\hline Cauda equina syndrome & $2(12)$ & $3(8)$ & \\
\hline \multicolumn{4}{|l|}{ Type of surgery (No. [\%]) } \\
\hline Anterior surgery & $6(35)$ & $16(43)$ & \multirow[t]{3}{*}{0.846} \\
\hline Posterior surgery & $7(41)$ & $7(19)$ & \\
\hline Combined surgery & $4(24)$ & $14(38)$ & \\
\hline \multicolumn{4}{|l|}{ Clinical outcome (No. [\%]) } \\
\hline Delayed wound healing & $1(6)$ & $7(19)$ & 0.411 \\
\hline Relapse & $1(6)$ & $10(27)$ & 0.143 \\
\hline Death & $3(18)$ & $5(14)$ & 0.7 \\
\hline \multicolumn{4}{|l|}{ Radiologic result (No. [\%]) } \\
\hline Solid fusion & $14(82)$ & $32(86)$ & 0.696 \\
\hline Segmental instability & 0 & 0 & \\
\hline
\end{tabular}

Data are presented as the mean \pm standard deviation or frequency (\%), *The difference is significant $(p<0.05)$, GNB gram-negative bacilli, GPC gram-positive cocci

Findings of magnetic resonance imaging

The MRI results showed that GNB-HPS patients had a significantly lower rate of epidural abscess compared with GPC-HPS patients ( $41 \%$ vs. $73 \%, p=0.024$ ) (Table 4 ).
Table 2 The history of infectious diseases between GNB-HPS and GPC-HPS

\begin{tabular}{llll}
\hline & $\begin{array}{l}\text { GNB-HPS } \\
(n=17)\end{array}$ & $\begin{array}{l}\text { GPC-HPS } \\
(n=37)\end{array}$ & $P$ \\
\hline History of infectious disease (No. [\%]) & $7(41)$ & $16(43)$ & 0.887 \\
Musculoskeletal infection (No. [\%]) & $2(12)$ & $11(30)$ & 0.189 \\
Cellulitis & $2(12)$ & $4(11)$ & \\
Necrotizing fasciitis & 0 & $5(14)$ & \\
Osteomyelitis & 0 & $2(5)$ & $0.03^{*}$ \\
Symptomatic urinary tract infection & $4(41)$ & $1(3)$ & \\
(No. [\%]) & & $1(3)$ & \\
Miscellaneous (No. [\%]) & $1(6)$ & $1(3)$ & \\
Pneumonia & $1(6)$ & 0 & \\
Port-A catheter infection & 0 & $1(3)$ \\
Liver abscess & 0 & 0 & \\
Infective endocarditis & $1(6)$ & \\
Periorbital abscess & 0 & \\
AV shunt infection & & &
\end{tabular}

*The difference is significant $(p<0.05)$, GNB-HPS gram-negative bacillary hematogenous pyogenic spondylodiscitis, GPC-HPS gram-positive coccal hematogenous pyogenic spondylodiscitis

Table 3 Microbiologic findings

\begin{tabular}{|c|c|}
\hline Microorganism & GNB $(n=17)$ \\
\hline \multicolumn{2}{|l|}{ Monomicrobial (No. [\%]) } \\
\hline Escherichia coli & $5(29)$ \\
\hline Enterobacter cloacae & $4(24)$ \\
\hline ESBL & $1(6)$ \\
\hline Klebsiella pneumonia & $2(12)$ \\
\hline Salmonella enteric & $2(12)$ \\
\hline Pseudomonas aeruginosa & $1(6)$ \\
\hline Proteus mirabilis & $1(6)$ \\
\hline Citrob.freundii & $1(6)$ \\
\hline \multicolumn{2}{|l|}{ Polymicrobial } \\
\hline Ps. aeruginosa + E.coli (ESBL) & $1(6)$ \\
\hline Microorganism (No. [\%]) & $\mathrm{GPC}(n=37)$ \\
\hline \multicolumn{2}{|l|}{ Monomicrobial (No. [\%]) } \\
\hline \multicolumn{2}{|l|}{ Staphylococcus aureus: } \\
\hline MSSA & $18(49)$ \\
\hline MRSA & $11(30)$ \\
\hline Coag(-) staphylococcus & $2(5)$ \\
\hline \multicolumn{2}{|l|}{ Streptococcus: } \\
\hline Group B Streptococcus & $2(5)$ \\
\hline Streptococcus viridans & $1(3)$ \\
\hline \multicolumn{2}{|l|}{ Polymicrobial } \\
\hline CONS, MSSA & $1(3)$ \\
\hline CONS, MRSA & $2(5)$ \\
\hline
\end{tabular}

ESBL Extended-spectrum $\beta$-lactamase, MSSA methicillin-sensitive Staphylococcus aureus, MRSA methicillin-resistant Staphylococcus aureus, CoNS Coag(-) staphylococcus 
Table 4 Findings of magnetic resonance imaging

\begin{tabular}{llll}
\hline Variable (No. [\%]) & GNB $(n=17)$ & GPC $(n=37)$ & $p$ \\
\hline Back muscle abscess & $6(35)$ & $11(30)$ & 0.683 \\
Epidural abscess & $7(41)$ & $27(73)$ & $0.024^{*}$ \\
Patient number in lumbar region & $n=15$ & $n=30$ & \\
$\quad$ Psoas muscle abscess & $12(80)$ & $21(70)$ & 0.755 \\
$\quad$ Single involvement & $3(25)$ & $6(29)$ & \\
$\quad$ Bilateral involvement & $9(75)$ & $15(71)$ & \\
\hline
\end{tabular}

*The difference is significant $(p<0.05)$

There were no significant differences in the incidence of psoas muscle abscess or back muscle abscess between GNB-HPS and GPC-HPS patients.

\section{Laboratory presentations}

There was no statistically difference in laboratory parameters between two group patients, even higher average levels of CRP and erythrocyte sedimentation rates in GPC-HPS patients ( $p=0.061$ and 0.076 , respectively) (Table 5). Microbial pathogens were isolated from the blood cultures of eight GNB-HPS patients and 23 GPCHPS patients ( $47 \%$ vs. $62 \%, p=0.297)$.

\section{Multivariate analysis of independent predicting risk factors} Independent predicting factors for GNB-HPS were a history of cancer (OR 57, 95\% CI 2-1563, $p=0.018$ ), and only constitutional symptoms versus only back pain (OR 9, 95\% CI 2.8-2666, $p=0.011$ ) (Table 6). An independent predicting factor for GPC-HPS was epidural abscess (OR 18, 95\% CI 1.4-250, $p=0.027$ ).

\section{Discussion}

Our results showed that compared with the 37 GPCHPS patients, the 17 GNB-HPS patients were more likely to be older individuals with a history of cancer. Chemotherapy/radiotherapy-induced alimentary mucositis occurs in patients treated for malignancy and the insufficient mucosal barrier contributes to enteric bacterial invasion and gram negative bacillary bacteremia with organisms such as Escherichia coli, Klebsiella, and Pseudomonas [9-12]. Numerous studies of bloodstream infection in the elderly indicated that gram-negative bacillary bacteremia was much more common in the

Table 5 Laboratory data

\begin{tabular}{llll}
\hline Variable & GNB $(n=17)$ & GPC $(n=37)$ & $p$ \\
\hline White blood cell count $\left(10^{3} / \mathrm{uL}\right)$ & $13.1 \pm 6.3$ & $13.8 \pm 4.8$ & 0.656 \\
Hemoglobin $(\mathrm{g} / \mathrm{dL})$ & $11.0 \pm 2.4$ & $11.7 \pm 2.6$ & 0.372 \\
C-reactive protein $(\mathrm{mg} / \mathrm{dL})$ & $110 \pm 57$ & $162 \pm 95$ & 0.061 \\
$\begin{array}{l}\text { Erythrocyte sedimentation rate } \\
\text { (mm/h) }\end{array}$ & $68.9 \pm 31.2$ & $84.3 \pm 25.9$ & 0.076 \\
Bacteremia (No. [\%]) & $8(47)$ & $23(62)$ & 0.297 \\
\hline
\end{tabular}

Table 6 Multivariate analysis of independent risk factors

\begin{tabular}{lll}
\hline Risk factor for GNB-HPS & $p$ value & OR $(95 \% \mathrm{Cl})$ \\
\hline Age & 0.479 & \\
Symptoms at the initial visit & $0.038^{*}$ & \\
Constitutional symptoms only/Back pain only & $0.011^{*}$ & $9(2.8-2666)$ \\
Malignancy & $0.018^{*}$ & $57(2-1563)$ \\
UTI & 0.122 & \\
Risk factor for GPC-HPS & & \\
$\quad$ Epidural abscess & $0.027^{*}$ & $18(1.4-250)$ \\
\hline *The difference is significant $(p<0.05)$ & &
\end{tabular}

elderly than in younger patients because the common infections in this population involve respiratory, urinary, and gastrointestinal systems [13-16]. A retrospective study of hematogenous vertebral osteomyelitis, conducted by Park, et al. [6], demonstrated that UTI was the main sources of infection in gram-negative vertebral osteomyelitis. Consistent with those results, age and UTI were significant factors for GNB-HPS in univariate analysis but not statistically predicting factor in multivariate analysis. The contributing reason may be that the incidence of cancer would increase with age and the high prevalence of UTI was found among the elderly.

Based on initial symptoms at first visit, this study showed that pain in the affected spinal region was the most common manifestation in GPC-HPS patients whereas constitutional symptoms (fever and drowsiness), with or without back pain, was the primary reason for initial physician visits in GNB-HPS patients. This latter finding may be attributed to be the much older age and frailty of our GNB-HPS patients. Clinical manifestations of spinal infection in aging or immunocompromised patients with cancer or poor physical function may affect their entire body with absence of localizing symptoms [17]. These patients may present with poor appetite, malaise, confusion, drowsiness, and fever. Therefore, GNB-HPS patients, who are more likely to have the early warning signs of drowsiness and fever, should seek immediate medical attention as, in contrast to patients with GPC infection, the majority of GNB-HPS patients in our study had acute onset of symptoms.

In this study, GPC-HPS possessed a higher incidence of epidural abscess formation than GNB-HPS. Staphylococcus aureus employs virulence factors to promote abscess formation [18, 19]. Similar to our findings, a comparative study concerning hematogenous vertebral osteomyelitis by GNB vs. methicillin-sensitive Staphylococcus aureus (MSSA) showed that patients with MSSA hematogenous vertebral osteomyelitis were more likely to have epidural abscess, and CRP values were higher in MSSA infection [6].

The overall mortality rate of our HPS patients at the 2-year follow-up was $14.8 \%$, which is similar to rates from previous studies which ranged from 1.5 to 
$38 \%[1,4,6,20,21]$. The large variance in these reported mortality rates may be attributed to different follow-up periods, varying in-hospital 6-month or 1-year mortality rates, and different causative microorganisms such as drug-resistant bacteria [6, 20, 21]. Park et al. reported an in-hospital mortality rate of $10 \%$, and the overall mortality rate was $20 \%$ within 1-year follow-up [22]. Kehrer et al. found that the mortality remained high in the first year after admission [23].

To date, there are no evidence-based guidelines addressing the best treatment methods in the management of pyogenic spondylodiscitis [24]. In review of literature, the recommended duration of antibiotic therapy is from 6 weeks to 3 months, including a 2-8 week course of intravenous antibiotics and a 6 week- 3 month course of oral antibiotics [24-26]. Although the mainstay of treatment for spondylodiscitis is long-term antibiotic therapy [25], surgical intervention is recommended in cases of spinal instability with vertebral destruction, abscess formation encroaching on psoas muscle or epidural spinal canal, and neurologic deficits.

Our study had several limitations including the small number of patients. Only a few patients were evaluated because we restricted HPS to a definitive diagnosis based on a microorganism isolated from the involved vertebrae in order to avoid the negative impact of misdiagnosis on the results. In addition, all patient data were collected from the Spine Operation Registry and HPS patients who received only antibiotic therapy were not included in this study. Combined antibiotic and surgical treatment was usually recommended in cases of deteriorated vertebral pathologic destruction, paraspinal abscess formation, and progressive sepsis. Although the characteristics of our patients may not be fully representative of all patients with HPS, the clinical features of patients in this study were consistent with those reported in the cohort studies of pyogenic spondylodiscitis, irrespective of surgical treatment [6].

\section{Conclusions}

GNB-HPS patients were more likely to have a higher prevalence of malignancy, and GPC-HPS patient had a higher incidence of epidural abscess. GNB-HPS patients had more-frequent constitutional symptoms and GPCHPS patients had predominant back pain. The clinical outcomes of GNB-HPS patients under combined surgical and antibiotic treatment were not different from those of GPC-HPS patients.

\section{Abbreviations}

CRP: C-reactive protein; GNB: Gram-negative bacilli; GNB-HPS: Gram-negative bacillary hematogenous pyogenic spondylodiscitis; GPC: Gram-positive cocci; GPC-HPS: Gram-positive coccal hematogenous pyogenic spondylodiscitis; HPS: Hematogenous pyogenic spondylodiscitis; MRI: Magnetic resonance imaging; UTI: Urinary tract infection

\section{Acknowledgments}

The authors thank the Research Committee of Chang Gung Memorial Hospital, Taiwan for assistance (No. CMRPG6F0131) in the Spine Operation Registry.

\section{Funding}

This study received no specific funding.

Availability of data and materials

Data used for analysis is available on request.

\section{Authors' contributions}

CYL conceived the study, participated in its design and drafted the manuscript. MHW collected data and helped to draft the manuscript. CCC participated in its design and intellectual input. TYH collected data. TJH participated in its design and intellectual input. YYL coordinated the research groups. JCH performed the statistical analysis. TJH and CYL participated in its design. All authors read and approved the final manuscript.

\section{Competing interests}

All authors declare that they have no competing interests.

\section{Consent for publication}

Not applicable.

\section{Ethics approval and consent to participate}

Our study was approved by our Ethics Committee and Institutional Review Board (IRB No. 103-2201B)

\section{Author details}

'Department of Orthopedic Surgery, Chang Gung Memorial Hospital, No. 6, West Sec., Chia Pu Rd., PuTz, Chiayi, Taiwan. 'Division of Infectious Diseases, Department of Internal Medicine, Chang Gung Memorial Hospital, Chiayi, Taiwan. ${ }^{3}$ Department of Ophthalmology, Chang Gung Memorial Hospital, Chiayi, Taiwan. ${ }^{4}$ Department of Nursing, Chang Gung University of Science and Technology, Chiayi, Taiwan. ${ }^{5}$ College of Medicine, Chang Gung University, Taoyuan, Taiwan. ${ }^{6}$ Department of Orthopedic Surgery, Taipei Medical University Hospital, Taipei, Taiwan. ${ }^{7}$ Graduate Institute of Clinical Medical Sciences, College of Medicine, Chang Gung University, Taoyuan, Taiwan.

Received: 15 February 2016 Accepted: 28 November 2016

Published online: 06 December 2016

\section{References}

1. Cottle L, Riordan T. Infectious spondylodiscitis. J Infect. 2008;56(6):401-12.

2. Hadjipavlou AG, Mader JT, Necessary JT, Muffoletto AJ. Hematogenous pyogenic spinal infections and their surgical management. Spine (Phila Pa 1976). 2000;25(13):1668-79.

3. Garcia Jr A, Grantham SA. Hematogenous pyogenic vertebral osteomyelitis. J Bone Joint Surg Am. 1960;42-A:429-36.

4. McHenry MC, Easley KA, Locker GA. Vertebral osteomyelitis: long-term outcome for 253 patients from 7 Cleveland-area hospitals. Clin Infect Dis. 2002;34(10):1342-50

5. Graham SM, Fishlock A, Millner P, Sandoe J. The management gram-negative bacterial haematogenous vertebral osteomyelitis: a case series of diagnosis, treatment and therapeutic outcomes. Eur Spine J. 2013;22(8):1845-53.

6. Park KH, Cho OH, Jung M, Suk KS, Lee JH, Park JS, Ryu KN, Kim SH, Lee SO, Choi $\mathrm{SH}$, et al. Clinical characteristics and outcomes of hematogenous vertebral osteomyelitis caused by gram-negative bacteria. J Infect. 2014;69(1):42-50.

7. Gouliouris T, Aliyu SH, Brown NM. Spondylodiscitis: update on diagnosis and management. J Antimicrob Chemother. 2010;65 Suppl 3:iii11-24.

8. Longo M, Granata F, Ricciardi K, Gaeta M, Blandino A. Contrast-enhanced MR imaging with fat suppression in adult-onset septic spondylodiscitis. Eur Radiol. 2003;13(3):626-37.

9. Minah GE, Rednor JL, Peterson DE, Overholser CD, Depaola LG, Suzuki JB. Oral succession of gram-negative bacilli in myelosuppressed cancer patients. J Clin Microbiol. 1986;24(2):210-3.

10. Napenas JJ, Brennan MT, Bahrani-Mougeot FK, Fox PC, Lockhart PB. Relationship between mucositis and changes in oral microflora during cancer chemotherapy. Oral Surg Oral Med Oral Pathol Oral Radiol Endod. 2007;103(1):48-59. 
11. Raber-Durlacher JE, Weijl NI, Abu Saris M, de Koning B, Zwinderman AH, Osanto S. Oral mucositis in patients treated with chemotherapy for solid tumors: a retrospective analysis of 150 cases. Support Care Cancer. 2000;8(5):366-71.

12. Wahlin $\mathrm{YB}$, Granstrom S, Persson S, Sjostrom M. Multivariate study of enterobacteria and Pseudomonas in saliva of patients with acute leukemia. Oral Surg Oral Med Oral Pathol. 1991;72(3):300-8.

13. Gavazzi G, Mallaret MR, Couturier P, Iffenecker A, Franco A. Bloodstream infection: differences between young-old, old, and old-old patients. J Am Geriatr Soc. 2002;50(10):1667-73.

14. McCue JD. Gram-negative bacillary bacteremia in the elderly: incidence, ecology, etiology, and mortality. J Am Geriatr Soc. 1987;35(3):213-8.

15. Meyers BR, Sherman E, Mendelson MH, Velasquez G, Srulevitch-Chin E, Hubbard M, Hirschman SZ. Bloodstream infections in the elderly. Am J Med. 1989;86(4):379-84

16. Whitelaw DA, Rayner BL, Willcox PA. Community-acquired bacteremia in the elderly: a prospective study of 121 cases. J Am Geriatr Soc. 1992; 40(10):996-1000.

17. Yoshikawa TT. Antimicrobial therapy for the elderly patient. J Am Geriatr Soc. 1990:38(12):1353-72.

18. Cheng AG, DeDent AC, Schneewind O, Missiakas D. A play in four acts: Staphylococcus aureus abscess formation. Trends Microbiol. 2011;19(5):225-32.

19. Cheng AG, Kim HK, Burts ML, Krausz T, Schneewind O, Missiakas DM. Genetic requirements for Staphylococcus aureus abscess formation and persistence in host tissues. FASEB J. 2009:23(10):3393-404.

20. Al-Nammari SS, Lucas JD, Lam KS. Hematogenous methicillin-resistant Staphylococcus aureus spondylodiscitis. Spine (Phila Pa 1976). 2007;32(22): 2480-6.

21. Pigrau C, Almirante B, Flores X, Falco V, Rodriguez D, Gasser I, Villanueva C, Pahissa A. Spontaneous pyogenic vertebral osteomyelitis and endocarditis: incidence, risk factors, and outcome. Am J Med. 2005;118(11):1287.

22. Park KH, Chong YP, Kim SH, Lee SO, Choi SH, Lee MS, Jeong JY, Woo JH, Kim YS. Clinical characteristics and therapeutic outcomes of hematogenous vertebral osteomyelitis caused by methicillin-resistant Staphylococcus aureus. J Infect. 2013;67(6):556-64

23. Kehrer M, Pedersen C, Jensen TG, Hallas J, Lassen AT. Increased short- and long-term mortality among patients with infectious spondylodiscitis compared with a reference population. Spine J. 2015;15(6):1233-40.

24. Shiban E, Janssen I, Wostrack M, Krieg SM, Horanin M, Stoffel M, Meyer B, Ringel F. Spondylodiscitis by drug-multiresistant bacteria: a single-center experience of 25 cases. Spine J. 2014;14(12):2826-34.

25. Zimmerli W. Clinical practice. Vertebral Osteomyelitis. N Engl J Med. 2010; 362(11):1022-9.

26. Bernard L, Dinh A, Ghout I, Simo D, Zeller V, Issartel B, Le Moing V, Belmatoug $\mathrm{N}$, Lesprit P, Bru JP, et al. Antibiotic treatment for 6 weeks versus 12 weeks in patients with pyogenic vertebral osteomyelitis: an open-label, non-inferiority, randomised, controlled trial. Lancet. 2015;385(9971):875-82.

\section{Submit your next manuscript to BioMed Centra and we will help you at every step:}

- We accept pre-submission inquiries

- Our selector tool helps you to find the most relevant journal

- We provide round the clock customer support

- Convenient online submission

- Thorough peer review

- Inclusion in PubMed and all major indexing services

- Maximum visibility for your research

Submit your manuscript at www.biomedcentral.com/submit

) Biomed Central 